\title{
$184-33332$
}

N/SN

Technical Memorandum $\mathbf{8 6 1 3 2}$

\section{THREE PRECISE GAMMA-RAY BURST SOURCE LOCATIONS}

T. L. Cline, U.D. Desai and B. J. Teegarden

C. Barat, K. Hurley, M. Niel and G. Vedrenne W. D. Evans, R. W. Klebesadel and J. G. Laros

I. V. Estulin, + A. V. Kuznetsov, V. M. Zenchenko and V. G. Kurt and B. Schaefer

JULY 1984

National Aeronautics and

Space Administration

Goddard Space Flight Center

Greenbelt. Maryland 20771 
T. L. Cline, U. D. Desai and B. J. Teegarden

Lab. for High Energy Astrophysics, NASA/GSFC, Greenbelt, MD, U.S.A.

C. Barat, K. Hurley, M. Niel and G. Vedrenne

Centre d'Etude Spatiale des Rayonnements (CNRS-UPS), Toulouse, FRANCE

W. D. Evans, R. W. Klebesadel and J. G. Laros

University of California, Los Alamos Scientific Lab., LoS Alamos, NM, U.S.A.

I. V. Estulin, ${ }^{+}$A. V. Kuznetsov, V. M. Zenchenko and V. G. Kurt

Institute for Space Research, Moscow, U.S.S.R.

and

B. E. Schaefer ${ }^{\star}$

Vept. of Physics and Ctr. for Space Research, M.I.T., Cambridge, MA, U.S.A.

\section{ABSTRACT}

The precise source regions of three moderately intense gamma ray bursts are derived. These events were observed with the first interplanetary burst sensor network on 1978 November 24 and 1979 November 5 and 16 . The optimum locations of the detectors, widely separated throughout the inner solar system; allowed for high-accuracy, over-determined source fields of size 0.7 to 7 . arc-min ${ }^{2}$. All three locations are at fairly high galactic latitude in reyions of low source confusion; none can be identified with a steady source object. A search in archived photographs for transients that can be associated with these source fields, however, produced one of the two optical flash events described in the companion Letter (Schaefer et al., 1984). These provide the first confirmation of the gamma-ray burst/optical transient association discovered by Schaefer (1981).

+ Deceased

* Present Address: University of Maryland and NASA/GSFC

** Accepted for publication by Astrophysical Journal (Letters) 


\section{INTRODUCT I DN}

The understandiny of yamma-ray bursts has been slow to evolve, due in larye part to the lack of identifications with known celestial objects. Only the event of 1979 March 5, a transient with characteristics that can he arguer to be anomalous (Cline et al., 1980), has a source field consistent with a knuwn celestial object. This is the supernova remnant N4G in the Large Mayellanic Cloud (Evans et al., 1980; Cline et al., 1942) which, as a source identification, is nevertheless controversial because of its extrayalactic distance. All other nigh-precision gamina-ray burst source fields are either eilluty of steddy optical objects down to may 22 (e.y., Larns et al., 1941; (iline et al., 1981; Barat et al., 1983) or can be subject to hackuround nhject. confusion (e.y., Barat et al., 1984). The discovery of an archived optical transient (Scnatefer, 1981), positioned within an apparently empty fieldof several arc min in dimension ( $\mathrm{Cl}$ ine et al., 19:1), may provide the means to reduce the search area to several arc-seconds in extent. Meep optical sedrches of this reyion have thus far produced evidence for source nhjects that are Ilaryinal, ambiyuous, and/or variable in time (Pederson et al., 198:3; schater et al., ly83).

We report here the accurately defined source fields of three additional yallmi-ray burst events detected with the first interplanetary network, the systell used for dll hiyh-precision directional measurements during la7k19830. These events may be the last that can be derived in such an optimum, over-determined manner (i.e., with four or more spacecraft at mutually yreat. separations--see Figure 1) until the late 1980)s. The three hursts vary in cnardcter but are all moderately intense--permitting their detailed study with the interplanetary network. The common feature resulting from the snurce 'trianyulations' is that of a precisely determined source field. All three 
are removed from the galactic disk and therefore in regions of relatively low stellar density. Searches for associated phenomena that can point to possible celestial source identifications are thus facilitated with these events. Two of the source fields are near to the celestial equator and are thus amenahle to scrutiny using yround-based telescopes in both the northern and southern nemispheres. The first and only known optical association with any of these reyions is an optical flash archived since 1901 , and recently uncovered, as described in the companion Letter (Schaefer et al., 1984). That paper reports on buth this and another optical flash (photographed in 1944) that appear to provide the first confirmation of the flash/burst association discovered hy Schaefer (1981). The original association linked a 1929, flash to the source ared (Cline et di., 1981) of the 1978 November 19 hurst. The lanl optical transient location is within the 1979 November 5 burst source region reported nere with d $\leq$ two standard-deviation error. This fit is entirely silitilar ton the fit of the initidlly discovered 1928 transient-1978 yamma-ray hurst source assuciation when analyzed in a similar inanner.

\section{INSTRUMENTATION}

The observations described here were made with various instrullents participating in the first interplanetary spacecraft network of yamma-ray Durst sensors. This array was composed of both satellites and spaceprohes. The near-Earth spacecraft included the same Vela satellites that discovered the yduma ray burst phenomenon over a decade ayo (Klebesadel et al., 1473), the Earth Satellite Proynoz-7 and the ISEE-3 probe. That vehicle orhited r.he. primary Sun-Earth Layranyian point when making the observatinns descrihed 
nere; it is now in a comet-encounter trajectory. The distant space probes were the Pioneer-Venus Orbiter, Veneras-11 and -12 in transit towards or away from Venus, and Helios-2 in solar orbit. A considerable number of other events are yet to be described in print by this consortium; these were observed either with fewer spacecraft and/or with less precision. Full descriptions of the instruments nave been published (see, e.g., Anderson et d1., 1978; Barat et al., 1981; Cline et al., 1979; Evans et al., 1979;

Klebesadel et al., 1980) and need no elaboration here. The Venera-11 and -1 ? and Proynoz-7 instruinents are Franco-Soviet collaborations on Soviet spacecraft. Tne Goddard Helios-2 instrument was added on to a Goddard experiment on the German Helios spacecraft and both the Los Alamos and the fordard ISFE-3 experiments were add-on modifications to other experiments on the NASA International Sun-Earth Explorer. The Pioneer-Venus Orhiter instrument is a Lus Aldmos experiment on that NASA vehicle, and LoS Alamos instruments on the Veld spacecraft array are independently supported, presently by the nepartment of Eneryy.

\section{DATA ANAL YSIS}

The data analysis techniques used to derive the gamina ray hurst snurce fields are a continuation of those methods described in earlier studies, such ds for the 1979 March 5 event (Evans et al., 1980; Cline et al., 198?), the ly79 April 6 event (Laros et al., 1981), the 1978 November 19 event (Cl ine et dl., 1981) and the 1979 June 13 event (Barat et al., 199.3). Analyses of those events nave demonstrated that the various residual errors in timiny accuracy are correctly estimated. The heliocentric timing technilue, which is entirely 
equivalent to an aberration correction, has also been developed. In this paper, we extend those methods with a new technique that derives a single, composite error field from four or more spacecraft observations. Mur previous derivations reported the most accurate, mutually-dependent three-spacecraft error fields (e.g., Evans et al., 1980 and Cline et al., 1981). The new Ilethod has the advantage that it makes use of all of the available directional infurmation, weighting each measurement appropriately to yield one final result. It can be outlined as follows. Comparison of the wavefront arrival times at each of any two spacecraft defines a ring-shaped celestial source reyion locus. The accuracy of this comparison is a function of how well the time nistories from the different detection instruments can he compared. (Figure 2 illustrates the variability in time-history determination for these events.) Any point in celestial coordinates is separated from each ring or annulus by some distance that is related to the probability of its likelihood ds source. Fur $N$ spacecraft there are $N(N-1) / 2$ annular loci that can mitually intersect in up to $N(N-1)(N-2) / 3$ source "triangulations". For example, five. spacecraft can produce 20 "triangulations". When the time history aliunment adjustments and readjustments are made (often with laborious reanalyses of data) such as to permit half of the intersections (one on each ring) to cluster, then the event is considered by the experimenters to be lncalized. Fur redundant, > 3-spacecraft situations, all the data producing these several overlapping 'trianyulations' are now taken into account by defining a youdness-of-fit value for each point in space. Given that the separation of that point from each of the source rings is associated with some error related to the mutual data comparison from that pair of observations, the normalizer rus summation of these errors constitutes the likelihond value of that point. ds suurce. The normalization results from the fact that 3 spacecraft can 
define one source field uniquely. Contours of regions of source likelihoor then result from plots of these grids of individual fit values.

\section{RESULTS}

The yamma-ray burst source fields are shown in figure 3 . The sizes of these source reyions vary from 0.7 to over 7 arc-inin?, sinall enough to easily perillit detailed study, particularly since they are all in regions of very low stellar imaye density. As yet, there is no evidence for association with any candidate steady source object. Computerized searches through catalnys of pusitions of compact objects including supernova remnants, pulsars, neutrnn stars and white dwarfs did not yield any positive results. This situation is similar to all the past burst event analyses, other than that of 1979 March b. An optical transient, similar to that discovered by Schaefer (1981), in the 1978 November 19 burst source field was, however, also found in the 1975 November $b$ burst source field developed here. The evidence for the existence of this optical event is discussed in a companion Letter (Schaefer et al., 1a84). This is one of two optical transients that provide the first confirmation of the ydillild-ray burst/optical transient association. The great advantage of the detection of an archived optical event is its aid in reducing the source location field by another order of maynitude or more, assuming no proper motion. veep optical scrutiny of the first of these three tiny $\left(\because 0.04\right.$ arc-inin $\left.{ }^{2}\right)$ source reyions has, thus far, produced only marginal and/or ambiguous results (Pederson et al., 1983; Schaefer et al., 1983).

This letter and a companion paper outlining the 1979 January 1.3 event. andlysis (Barat et al., 1984) complete the published descriptions of all the 
most accurately definable source fields from the first network that are overdetermined, i.e., all those that can be determined with a wide, interplanetary scatter of 24 spacecraft. Descriptions of certain other welldefined burst source reyions are in press (Laros et al., 1984). These events occurred after the Venera spacecraft separated at a yreat distance from Venus (and from the Earth), providing a third long baseline, required after the demise of Helios-2. Future high-precision yanma ray hurst source location ubservatiuns from interplanetary spacecraft will also include some events from the second network, making use of observations from Veneras-13 and -14 during a severdl-month period with adequately long baselines following their Venus encounters in 1982-1983. The ICE (formerly called ISEF-3) spacecraft in its 1984-1985 comet-encounter trajectory may make possible an additional longDaseline network of limited lifespan. Finally, networks later in the decare. will incorporate the International Solar Polar Missinn and possibly other planetary missions. It is yet entirely possible that some source pattern or identification will emerge from their results and from the optical transient. studies they promote. 


\section{REFERENCES}

Anderson, K. A., et al. 1978, IEEE Trans., GE-16, 157.

Bdrat, C., et al., 1984 (Ap. J., in press).

Barat et al., (submitted to Ap. J. Letters).

Cline, T., et al., 1979, Ap. J. (Letters) 229, L47.

Cline, T., et al., 1980, Ap. J. (Letters), 237, L1.

Cline, T., et al., 1981, Ap. J. (Letters), 246, L133.

Cline, T., et al., 1982, Ap. J. (Letters), 255, L45.

Evans, W. D., et al., 1980, Ap. J. (Letters), 237, L7.

Klebesadel, R. W., Strong, I. B., and 0lson, R. A., 1973, Ap. J. (Letters), 182, $L 85$.

Klebesadel, R., Evans, W., Glore, J., Spalding, R., and Wymer, F., 1980, IF.F.E

Trans. GE-18, 1 .

Laros, J. G., et al., 1981, Ap. J. (Letters), 245, L63.

Laros, J. G., et al., 1984, submitted to Ap. J.

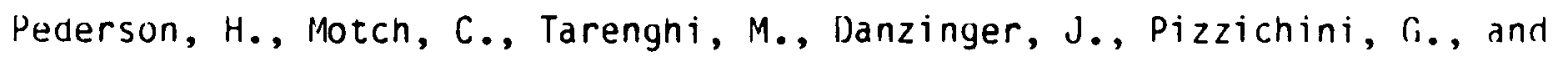
Lewin, W. H. G., 1983, Ap. J. (Letters) 270, L43.

Schdefer, B. E., 1981, Nature, 294, 722.

Schaefer, B. E., Seitzer, P., Bradt, H. V., 1983, Ap. J. (Letters), 270, L49. Schaefer, B., et al., 1984, submitted to Ap. J. (Letters).

FIGURE CAPTIONS

Figure 1. Instrument yeometry for the three burst events projected on the ecliptic plane: the 1979 November arrays (t and $x$ ) constitute optimum detector situations with overdeterminations (> 3 spacecraft) having mutual separations all with long baselines. The 1978 
November array is more typical, with a fractional-All scatter (indicated with dots). The Earth-orbiters Prognoz-7 and Vela 5 and the position of the Sun give the 1-AU scale. The ecliptic latitudes of the 3 gamma-ray burst directional vectors are 15 to 2 ? degrees. This fact, combined with the near-ecliptic plane locations of the instruments, limits the source field accuracies more in declination than in right ascension.

Figures $2 a, b$, and $c$. Sample time histories of the bursts. (a), the 1979 November 16 event is statistically well defined; it is unusual with a quiet gap of nearly 1-minute duration separating two intense features. (b), the November 24 event consists of broader, less well-defined features. (c), the November 5 event is fairly weak but typical, with randomly and rapidly fluctuating features. The accuracies of time history comparison vary, with typical values in the 50-120 insec range.

Figures $3 a, b$, and $c$. The contours of source region likelihnod: the dashed areas are meant to represent 99 percent confidence linits, and the inner areas, 90 percent confidence. The error field of the best resolved event is about 0.7 arc- $\min { }^{2}$ in size. The source of the optical transient of Schaefer (1984) is located less than 1 arc-minute from the axis of one burst source field, but is not entirely inside the inner area. This is taken to be statistically reasonable and is not regarded as meaningful evidence for source proper motion. A reanalysis, with this method, of the 1978 November 19 burst event gives the location of the first archived optical flash (Schaefer, 1981) also to be on the edge of the inner, $\sim 90$ percent-confident, area. 
ACKNOWLEDGEMENTS We wish to thank several persons for their helpful suygestions with the manuscript, especially E. Fenimore and the Referee. The French work was supported by CNES Contract No. 79212. The effort at Los Aldmos was carried out under the aupices of the U. S. Department of Fnergy and NASA, under Contract No. 98331A.

\section{AUUKESSES}

T. L. Cline, U. D. Uesai, B. E. Schaefer, and B. J. Teegarden: NASA/foddard Space Flight Center, Code 661, Greenbelt, MD 20771.

C. Barat, K. Hurley, M. Niel, and G. Vedrenne: Centre d'Etude Spatiale des Rayonnements, B. P. 4346, 31029 Toulouse Cedex, France.

W. U. Evans, R. W. Klebesadel, and J. G.'Laros: Los Alamos National Laboratory, Los Alamos, NM 87544.

I. V. Estulin: Deceased

A. V. Kuznetsov, V. M. Zenchenko, and V. G. Kurt: Institute for Space Researcn (IKI), Profsoyouznaya 88, 117810 Moscow, II.S.S.R. 

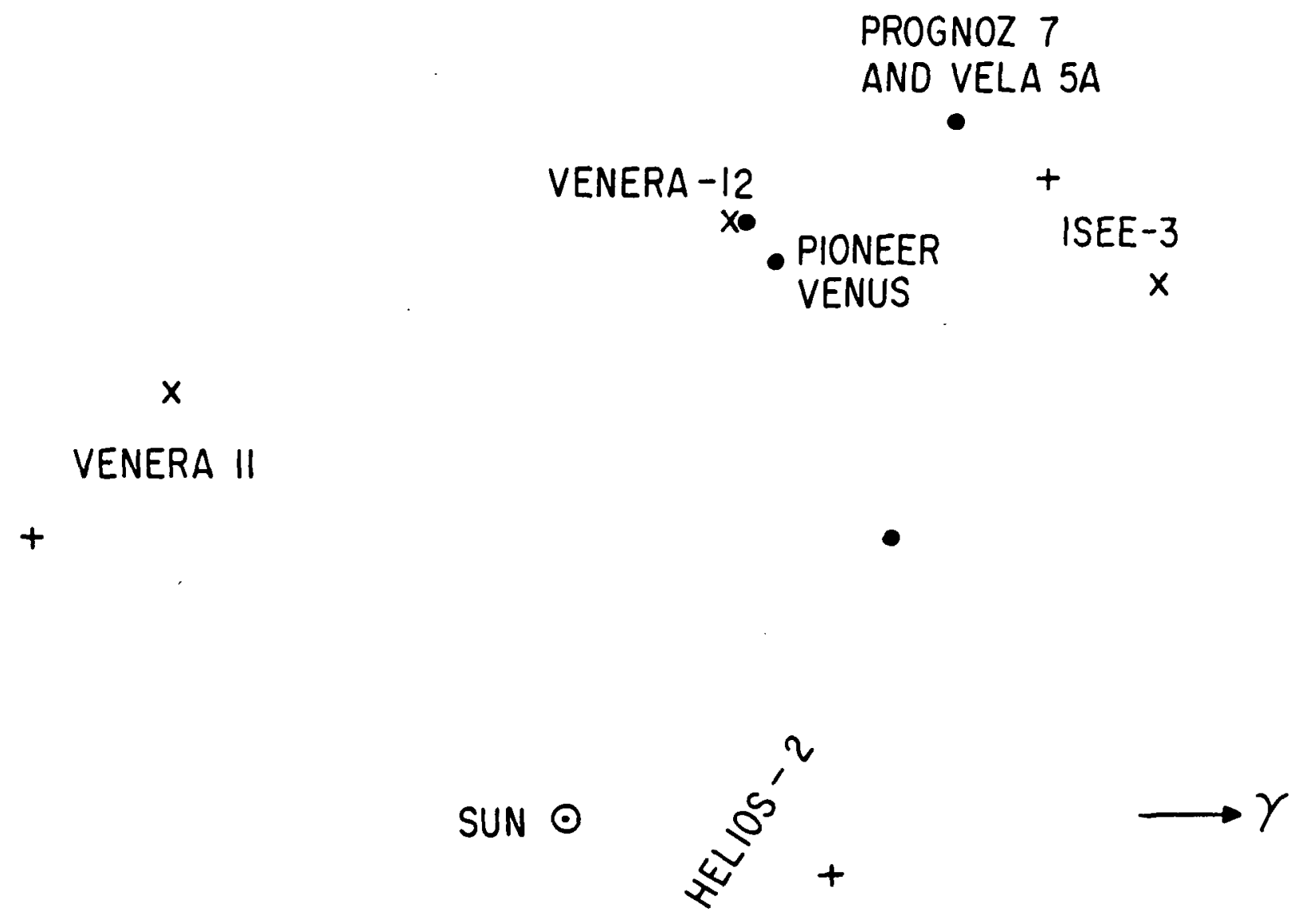

- 78 NOV 24

$\times \quad 79$ NOV 5

+79 NOV 16

$x$

PIONEER VENUS ORBITER
$x$

$5 i .1$ 


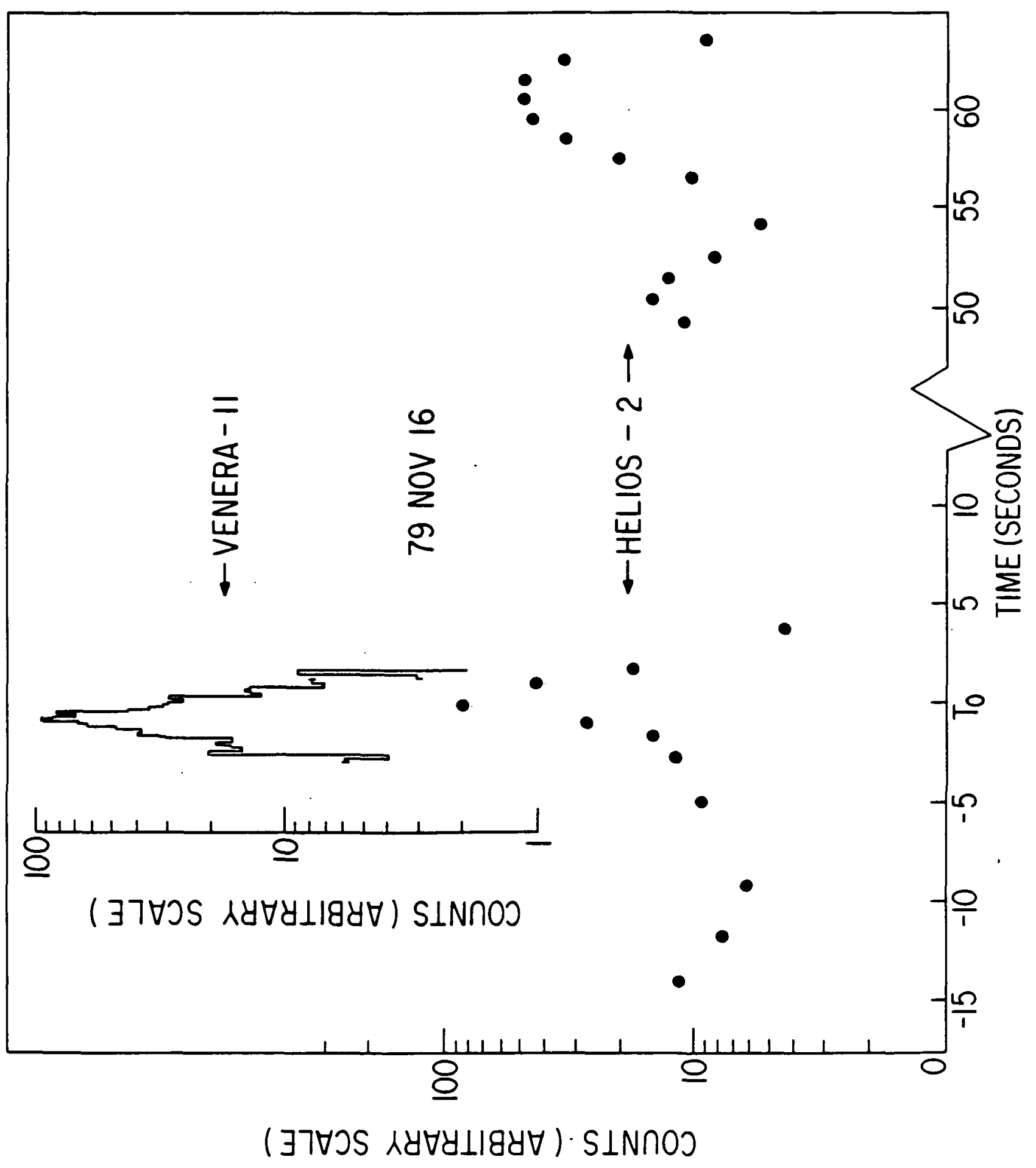

Fig. 2A 

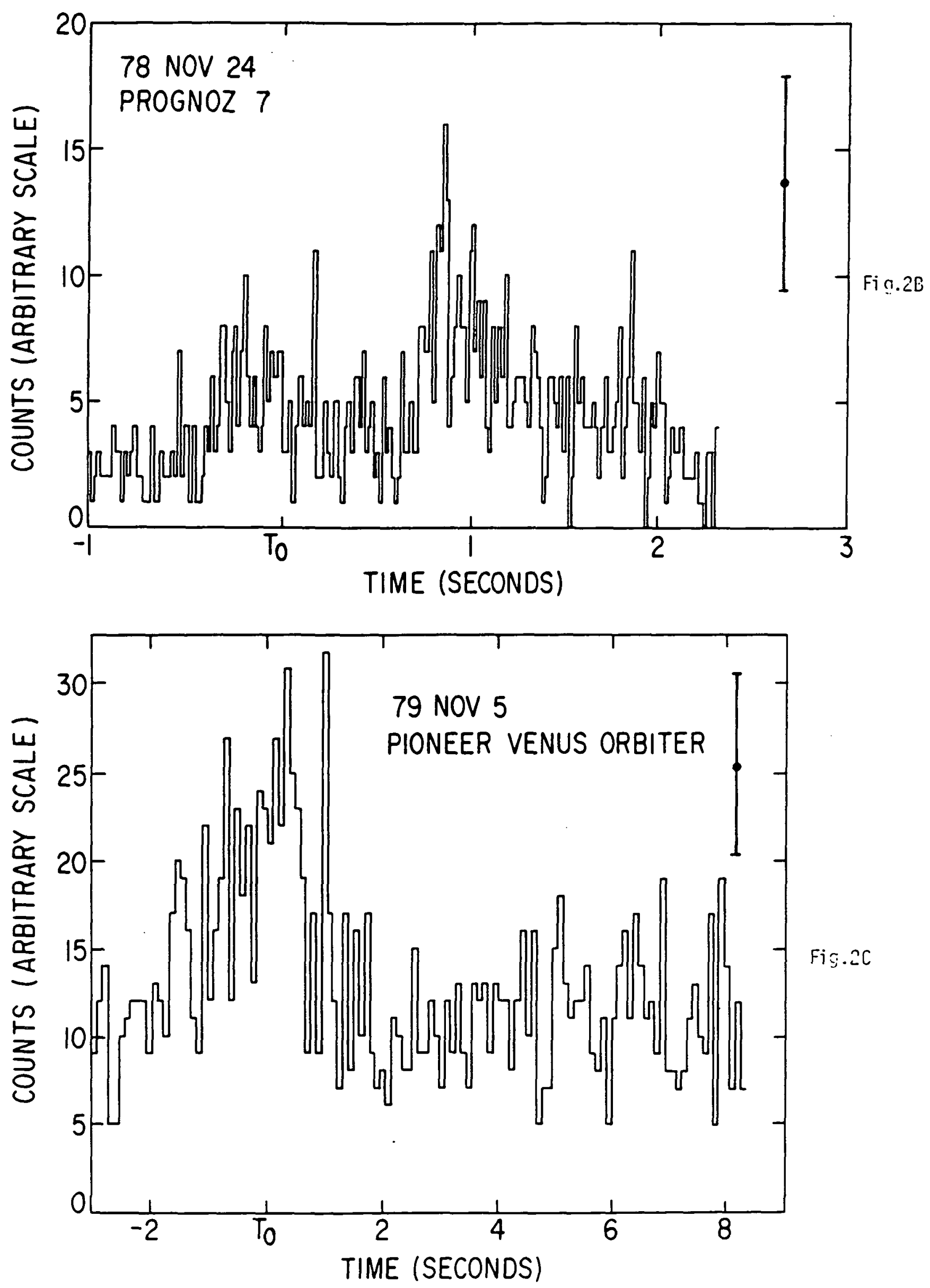


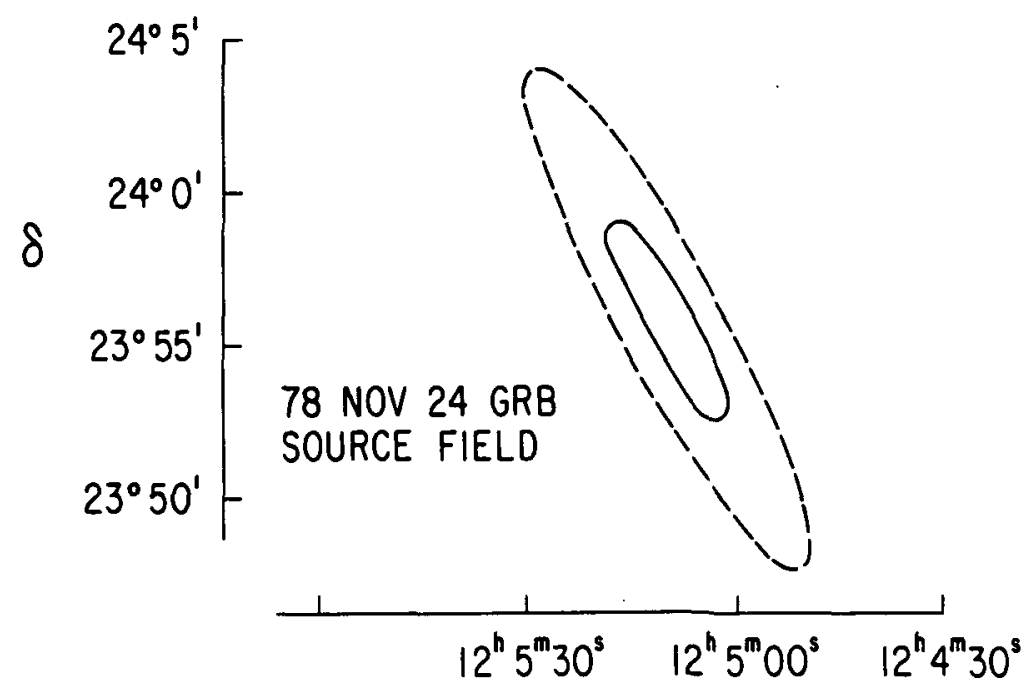

Fig. $3 \hat{n}$
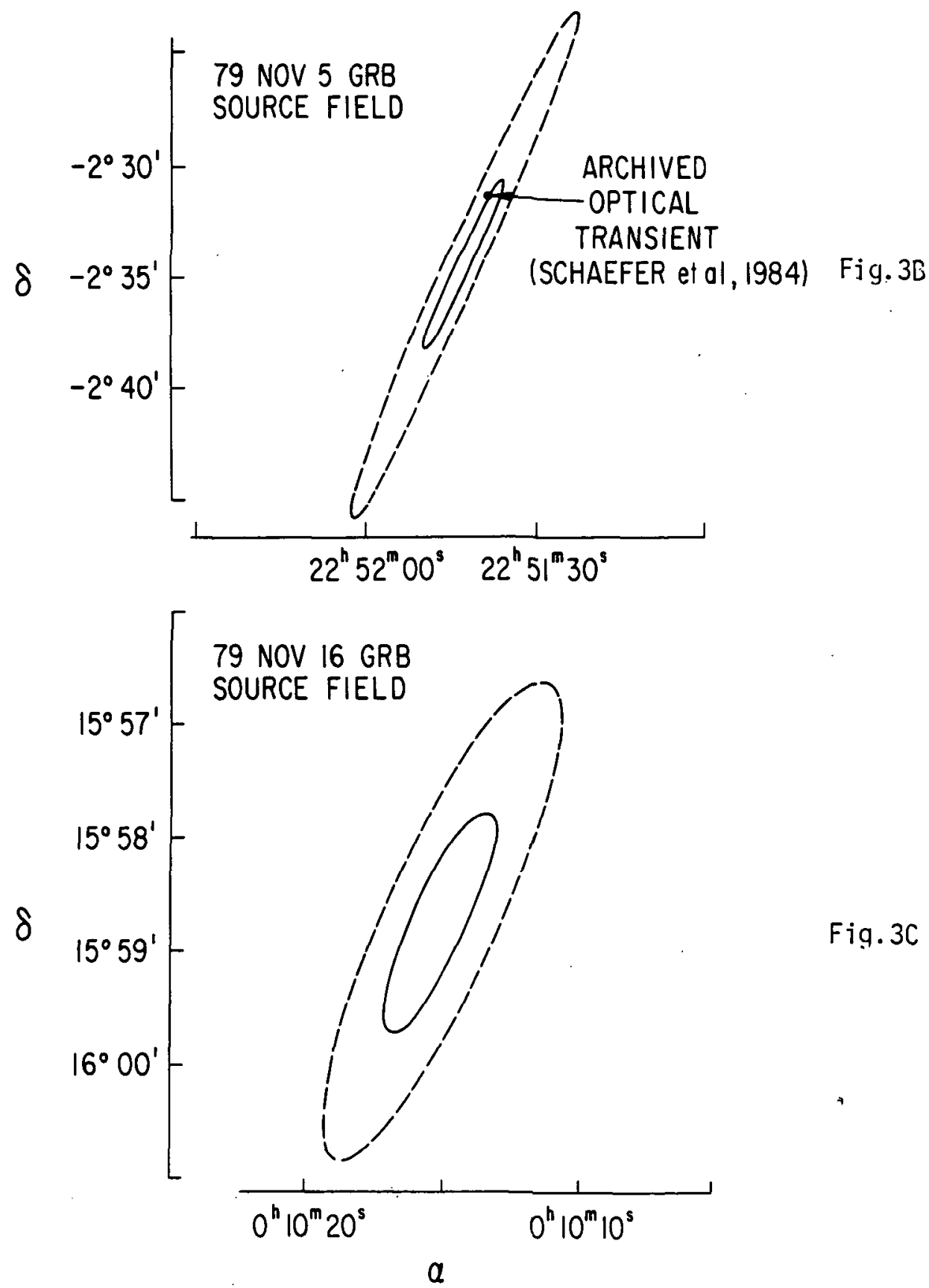

Fig. 3C 
BIBLIOGRAPHIC DATA SHEET

\begin{tabular}{|c|c|}
\hline $\begin{array}{l}\text { 1. Report No. } \\
\text { TM-86132 }\end{array}$ & 3. Recipient's Catalog No. \\
\hline \multirow{2}{*}{$\begin{array}{l}\text { 4. Title and Subtitle } \\
\text { THREE PRECISE GAMMA-RAY BURST SOURCE LOCATIONS }\end{array}$} & $\begin{array}{l}\text { 5. Report Date } \\
\text { July } 1984\end{array}$ \\
\hline & $\begin{array}{l}\text { 6. Performing Organization Code } \\
660\end{array}$ \\
\hline 7. Author(s) & 8. Performing Organization Report No. \\
\hline \multirow{3}{*}{$\begin{array}{l}\text { 9. Performing Organization Name and Address } \\
\text { Laboratory for High Energy Astrophysics }\end{array}$} & 10. Work Unit No. \\
\hline & 11. Contract or Grant No. \\
\hline & 13. Type of Report and Period Covered \\
\hline \multirow[t]{2}{*}{ 12. Sponsoring Agency Name and Address } & \\
\hline & 14. Sponsoring Agency Code \\
\hline
\end{tabular}

15. Supplementary Notes

To be published in Astrophysical Journal (Letters)

16. Abstract

The precise source regions of three moderately intense gamma-ray bursts are derived. These events were observed with the first interplanetary burst sensor network on 1978 November 24 and 1979 November 5 and 16. The optimum locations of the detectors, widely separated throughout the inner solar system, allowed for high-accuracy, over-determined source fields of size 0.7 to 7 . arc-min2. All three locations are at fairly high galactic latitude in regions of low source confusion; none can be identified with a steady source object. A search in archived photographs for transients that can be associated with these source fields, however, produced one of the two optical flash events described in the companion Letter (Schaefer et a1., 1984). These provide the first confirmation of the gamma-ray burst/optical transient association discovered by Schaefer (1981).

17. Key Words (Selected by Author(s))

18. Distribution Statement

Gamma-rays, Transients

19. Security Classif. (of this report)

UN

20. Security Classif. (of this page)

UN

21. No. of Pages

22. Price ${ }^{*}$

16

"For sale by the National Technical Information Service. Springfield, Virginia 